\title{
Three-dimensional propagation of the transmitted shock wave in a square cross-sectional chamber
}

\author{
Z. Jiang ${ }^{1}$, C. Wang ${ }^{1}$, Y. Miura ${ }^{2}$, K. Takayama ${ }^{3}$ \\ 1 LHD, Institute of Mechanics, Chinese Academy of Sciences, Beijing 100080, P.R. China \\ 2 Department of Sciences, Faculty of Sciences, Yamaguchi Unversity, Yoshida 1677-1, Yamaguchi 753-8512, Japan \\ 3 Shock Wave Research Center, Institute of Fluid Science, Tohoku University, 2-1-1 Katahira, Aoba-ku, Sendai 980-8577, Japan
}

Received 6 July 2002 / Accepted 24 May 2003

Published online 4 July 2003 - (c) Springer-Verlag 2003

Communicated by Z.-Y. Han

\begin{abstract}
An investigation into the three-dimensional propagation of the transmitted shock wave in a square cross-section chamber was described in this paper, and the work was carried out numerically by solving the Euler equations with a dispersion-controlled scheme. Computational images were constructed from the density distribution of the transmitted shock wave discharging from the open end of the square shock tube and compared directly with holographic interferograms available for CFD validation. Two cases of the transmitted shock wave propagating at different Mach numbers in the same geometry were simulated. A special shock reflection system near the corner of the square cross-section chamber was observed, consisting of four shock waves: the transmitted shock wave, two reflection shock waves and a Mach stem. A contact surface may appear in the four-shock system when the transmitted shock wave becomes stronger. Both the secondary shock wave and the primary vortex loop are three-dimensional in the present case due to the non-uniform flow expansion behind the transmitted shock.
\end{abstract}

Key words: Shock waves, Mach reflection, Numerical simulation, CFD validation

PACS: $43.40 . \mathrm{Nm}$

\section{Introduction}

Shock wave propagation in two-dimensional cases has been well investigated in the last several decades because of its important applications, particularly for example, shock wave reflections over wedges (Hornung 1986, Ben-Dor 1992), shock wave diffractions around sharp corners (Skews 1967, Takayama and Inoue 1991) and shock wave focusing in specially-designed reflectors (Terao 1984, Grönig 1989). The pioneer research led to good understanding on two-dimensional shock wave propagation and reflection, but little work has been reported so far on the propagation of three-dimensional shock waves, which is of more importance in engineering. This is because threedimensional shock wave phenomena are somewhat difficult to visualize clearly by using flow visualization techniques. Moreover, the visualized images of the shock waves are very complicated and difficult to interpret due to the three-dimensional effects occurring in flow visualization process.

As is well known, with development of high resolution shock-capturing schemes in the research area of Computational Fluid Dynamics (CFD), numerical simulations be-

Correspondence to: Z. Jiang (e-mail: zljiang@imech.ac.cn) come a quite powerful tool to highlight flow physics in three-dimensional complex flowfields. However, numerical solutions must be validated before the results are convinced to describe the physics correctly because the governing equations, initial and boundary conditions used are only approximations, to a certain extent, of the conservation laws of fluid flows. This leads to another problem, that is, the validation of numerical simulations with experiments. Such validation for shock wave research requires the comparison of the numerical solutions of the entire flowfield with experimental data measured by nonintrusive techniques at the same instant. The topic is still a challenging problem in CFD because the threedimensional flowfields, often including shock wave reflection, diffraction and interaction, are always highly transient. On the other hand, the experimental data from flow visualization also need to be reliably confirmed and carefully interpreted with the help of numerical simulations because of the complex nature of the three-dimensional flows.

One of the three-dimensional shock waves possibly created in the laboratory with shock tubes is the shock wave diffracting at the open-end of a square cross-section tube. The transmitted shock wave, at its initial stage, is planar, but quickly deforms into a three-dimensional con- 


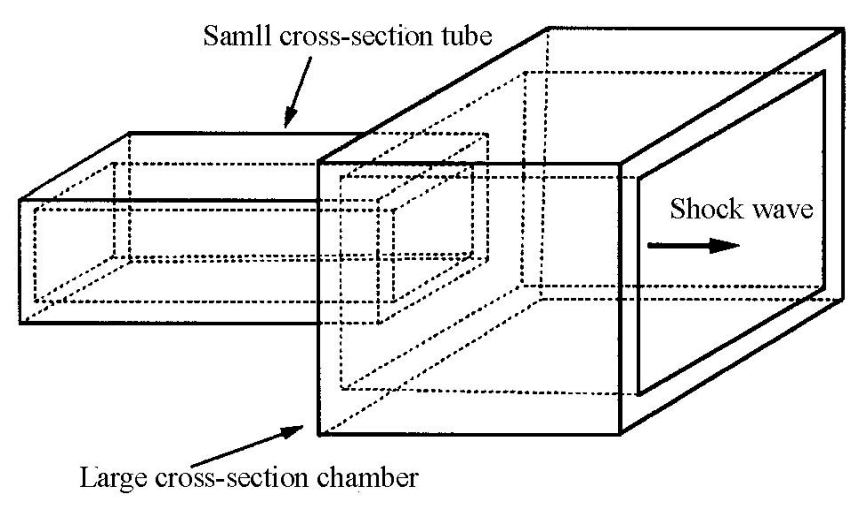

Fig. 1. Computational domain for numerical simulation of three-dimensional shock wave propagation

figuration as it propagates. This shock wave was investigated earlier by Abe and Takayama (1989) and then by Watanabe and Takayama (1992) using double exposure holographic interferometry. In their work, the shock wave propagating at a Mach number of $M_{s}=1.5$ in air was visualized from the direction normal to that of the shock wave propagation. The transmitted shock wave and the primary vortex loop behind it were clearly observed from their experiments. Further understanding came from the work reported by Jiang et al. (1999). The motion of the transmitted shock wave and the primary vortex loop behind it were well demonstrated with the combination of numerical and experimental results. Investigation into the three-dimensional shock reflection was reported by Meguro et al. (1997). In their study, a planar shock wave propagates from a square cross-section shock tube into a converging chamber with a special corner consisting of two wedges. The results obtained were compared with the analytic data of two-dimensional shock wave reflection, and some similar characteristics were reported. A special test case for investigating three-dimensional shock wave propagation is proposed in this paper. It is the transmitted shock wave propagating in a specially designed test section as shown in Fig. 1, where a small cross-section shock tube is connected to a large cross-section chamber. The transmitted shock wave is planar while it propagates in the shock tube, but diffracts quickly into a three-dimensional configuration after it discharges into the chamber. This transmitted shock will reflect, first from the middle of chamber walls and then from the chamber corners. Truly three-dimensional wave phenomena will be developed in the geometric domain, and is a useful case for the fundamental study on three-dimensional shock propagation.

The objective of this paper is to investigate the threedimensional shock wave propagation and reflection. The Euler equations were chosen as the governing equations and solved using the dispersion-controlled scheme (Jiang 1993 and Jiang et al. 1995). The pioneer work has demonstrated that the viscous effect on shock wave propagation and reflection is negligible, but it will have some effects on the beginning process of the shock-wave/primary-vortex interaction. Moreover, viscosity plays an important role in the vortex break-down process of the later stage of the interaction. Considering the objective and the above discussion, we validated numerical solutions with experimental results obtained by using holographic interferometry to demonstrate its reliability, and focused our discussion on the three-dimensional shock wave propagation, reflection and diffraction. The shock/vortex interaction at its initial stage will be mentioned conceptually, since it is believed that the beginning process of the interaction may be affected more or less if measured in flow variables, but the main configuration of the shock/vortex interaction will remain unchanged. The vortex break-down and turbulence generation will be left for future study where viscosity and turbulence models must be included in numerical simulations.

\section{Governing equations and numerical methods}

Assuming that the flow field in the present study is symmetrical and viscosity effects on shock wave propagation are negligible, a hyperbolic system of three-dimensional conservation laws for a perfect gas in Cartesian coordinates can be written as

$$
\frac{\partial \mathbf{U}}{\partial t}+\frac{\partial \mathbf{F}}{\partial x}+\frac{\partial \mathbf{G}}{\partial y}+\frac{\partial \mathbf{E}}{\partial z}=0
$$

where $\mathbf{U}, \mathbf{F}, \mathbf{G}$ and $\mathbf{E}$ denote the state variables and fluxes in $x-, y-$ and $z$-directions, respectively, given by

$$
\begin{array}{r}
\mathbf{U}=\left(\begin{array}{c}
\rho \\
\rho u \\
\rho v \\
\rho w \\
e
\end{array}\right), \quad \mathbf{F}=\left(\begin{array}{c}
\rho u \\
\rho u^{2}+p \\
\rho u v \\
\rho u w \\
(e+p) u
\end{array}\right), \\
\mathbf{G}=\left(\begin{array}{c}
\rho v \\
\rho v u \\
\rho v^{2}+p \\
\rho v w \\
(e+p) v
\end{array}\right), \quad \mathbf{E}=\left(\begin{array}{c}
\rho w \\
\rho w u \\
\rho w v \\
\rho w^{2}+p \\
(e+p) w
\end{array}\right),
\end{array}
$$

where the primitive variables in the unknown $\mathbf{U}$ are density $\rho$, velocity components $u, v$ and $w$, respectively. $p$ is the fluid pressure and $e$, the total energy per unit volume, is related to the equation of state for perfect gas given by

$$
e=\frac{p}{\gamma-1}+\frac{1}{2}\left(u^{2}+v^{2}+w^{2}\right)
$$

where $\gamma$, the specific heat ratio, is taken as 1.4 for air in the present numerical simulations.

The explicit difference equations of Eq. (1) discretized in space using the dispersion-controlled scheme (Jiang et al. 1995) are given in the form of half discretization as

$$
\begin{aligned}
\left(\frac{\partial \mathbf{U}}{\partial t}\right)_{i, j, k}^{n} & \\
= & -\frac{1}{\Delta x}\left(\mathbf{H}_{i+\frac{1}{2}, j, k}^{n}-\mathbf{H}_{i-\frac{1}{2}, j, k}^{n}\right)-\frac{1}{\Delta y}\left(\mathbf{P}_{i, j+\frac{1}{2}, k}^{n}-\mathbf{P}_{i, j-\frac{1}{2}, k}^{n}\right) \\
& -\frac{1}{\Delta z}\left(\mathbf{Q}_{i, j, k+\frac{1}{2}}^{n}-\mathbf{Q}_{i, j, k-\frac{1}{2}}^{n}\right)
\end{aligned}
$$


with

$$
\left\{\begin{array}{l}
\mathbf{H}_{i+\frac{1}{2}, j, k}^{n}=\mathbf{F}_{i+\frac{1}{2} L, j, k}^{+}+\mathbf{F}_{i+\frac{1}{2} R, j, k}^{-} \\
\mathbf{P}_{i, j+\frac{1}{2}, k}^{n}=\mathbf{G}_{i, j+\frac{1}{2} L, k}^{+}+\mathbf{G}_{i, j+\frac{1}{2} R, k}^{-} \\
\mathbf{Q}_{i, j, k+\frac{1}{2}}^{n}=\mathbf{E}_{i, j, k+\frac{1}{2} L}^{+}+\mathbf{E}_{i, j, k+\frac{1}{2} R}^{-}
\end{array}\right.
$$

where

$$
\begin{aligned}
& \left\{\begin{array}{l}
\begin{array}{l}
\mathbf{F}_{i+\frac{1}{2} L, j, k}^{+} \\
=\mathbf{F}_{i, j, k}^{+}
\end{array}+\frac{1}{2} \mathbf{\Phi}_{\mathbf{A}}^{+} \operatorname{minimod}\left(\Delta \mathbf{F}_{i-\frac{1}{2}, j, k}^{+}, \Delta \mathbf{F}_{i+\frac{1}{2}, j, k}^{+}\right) \\
\mathbf{F}_{i+\frac{1}{2} R, j, k}^{-} \\
=\mathbf{F}_{i+1, j, k}^{-}-\frac{1}{2} \boldsymbol{\Phi}_{\mathbf{A}}^{-} \operatorname{minimod}\left(\Delta \mathbf{F}_{i+\frac{1}{2}, j, k}^{-}, \Delta \mathbf{F}_{i+\frac{3}{2}, j, k}^{-}\right),
\end{array}\right. \\
& \left\{\begin{array}{c}
\mathbf{G}_{i, j+\frac{1}{2} L, k}^{+} \\
=\mathbf{G}_{i, j, k}^{+}+\frac{1}{2} \mathbf{\Phi}_{\mathbf{B}}^{+} \operatorname{minimod}\left(\Delta \mathbf{G}_{i, j-\frac{1}{2}, k}^{+}, \Delta \mathbf{G}_{i, j+\frac{1}{2}, k}^{+}\right) \\
\mathbf{G}_{i, j+\frac{1}{2} R, k}^{-} \\
=\mathbf{G}_{i, j+1, k}^{-}-\frac{1}{2} \mathbf{\Phi}_{\mathbf{B}}^{-} \operatorname{minimod}\left(\Delta \mathbf{G}_{i, j+\frac{1}{2}, k}^{-}, \Delta \mathbf{G}_{i, j+\frac{3}{2}, k}^{-}\right),
\end{array}\right.
\end{aligned}
$$$$
\left\{\begin{array}{l}
\mathbf{E}_{i, j, k+\frac{1}{2} L}^{+} \\
\quad=\mathbf{E}_{i, j, k}^{+}+\frac{1}{2} \boldsymbol{\Phi}_{\mathbf{C}}^{+} \operatorname{minimod}\left(\Delta \mathbf{E}_{i, j, k-\frac{1}{2}}^{+}, \Delta \mathbf{E}_{i, j, k+\frac{1}{2}}^{+}\right) \\
\mathbf{E}_{i, j, k+\frac{1}{2} R}^{-} \\
\quad=\mathbf{E}_{i, j, k+1}^{-}-\frac{1}{2} \mathbf{\Phi}_{\mathbf{C}}^{-} \operatorname{minimod}\left(\Delta \mathbf{E}_{i, j, k+\frac{1}{2}}^{-}, \Delta \mathbf{E}_{i, j, k+\frac{3}{2}}^{-}\right)
\end{array}\right.
$$$$
\left\{\begin{array}{l}
\Delta \mathbf{F}_{i+\frac{1}{2}, j, k}^{ \pm}=\mathbf{F}_{i+1, j, k}^{ \pm}-\mathbf{F}_{i, j, k}^{ \pm} \\
\Delta \mathbf{G}_{i, j+\frac{1}{2}, k}^{ \pm}=\mathbf{G}_{i, j+1, k}^{ \pm}-\mathbf{G}_{i, j, k}^{ \pm} \\
\Delta \mathbf{E}_{i, j, k+\frac{1}{2}}^{ \pm}=\mathbf{E}_{i, j, k+1}^{ \pm}-\mathbf{E}_{i, j, k}^{ \pm}
\end{array}\right.
$$

Fig. 2a,b. Side views of the transmitted shock wave diffracting near the open end of a square shock tube at a Mach number of $M_{i}=1.5$. a Numerical interferogram; $\mathbf{b}$ experimental interferogram

shown in Fig. 1 needs to be calculated. Reflecting boundary conditions were specified both on solid walls and the planes of symmetry. Non-reflecting boundary conditions were applied at the inflow and outflow boundaries. The equally-spaced grid system of $600 \times 110 \times 110$ mesh points was used and the Courant number was taken as 0.5 in all the numerical simulations.

\section{Validation of numerical algorithms}

In order to verify the numerical algorithms and validate the numerical solutions, a transmitted shock wave discharging from the open end of a square cross-section shock tube into ambient air at a Mach number of $M_{s}=1.5$ was simulated. The geometry of the open end is similar to the one shown in Fig. 1, but without the large cross-section chamber. The shock wave diffraction here is the same as that to be investigated before the transmitted shock wave is reflected from the chamber wall. Experiments were conducted in a $40 \mathrm{~mm} \times 40 \mathrm{~mm}$ square cross-section tube being $840 \mathrm{~mm}$ long, which is connected to a $60 \mathrm{~mm} \times 150 \mathrm{~mm}$ diaphragmless shock tube in the Shock Wave Research Center, Tohoku University, Japan. The diffraction of the transmitted shock wave was visualized from three viewing directions with double exposure holographic interferometry (Casey and Takayama 1991, Jiang et al. 1999).

By integrating the three-dimensional density distribution along light rays, computational interferograms were constructed for direct comparison with experimental interferograms (Jiang and Takayama 1999). Numerical and experimental results viewed from three viewing directions 


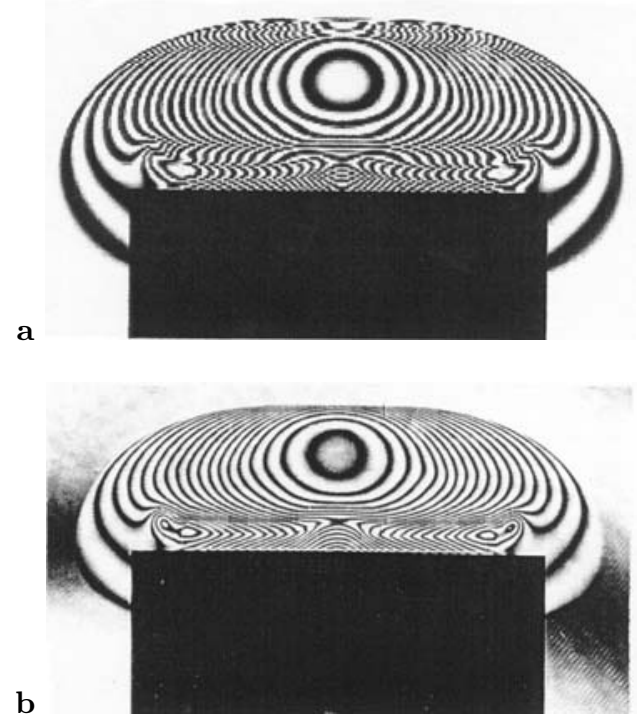

Fig. 3a,b. Corner views of the transmitted shock wave diffracting near the open end of a square shock tube at a Mach number of $M_{i}=1.5$. a Numerical interferogram; b Experimental interferogram

were given from Figs. 2 to 4 . Figure 2 shows the side view of the shock wave diffraction, which is obtained by viewing from the direction normal to the side-wall of the shock tube. Figure 3 shows the corner view that is created by viewing along the diagonal line of the chamber crosssection. Figure 4 shows the axial view from a direction of $15^{\circ}$ off the axis of symmetry.

Upon carefully examining each pair of interferograms shown from Figs. 2 to 4 , it is seen that the agreement between the numerical results and the experimental data is excellent because of the fact that the number of fringes and their distributions coincide very well with each other in all the three pairs of flow images. The discrepancy observable between the numerical and experimental interferograms is only near the exit in Figs. 2a and 3a. This is due to the lack of the resolution for displaying these numerical images, that is, there are not enough pixels in the computed images to distinguish as many fringes as in the experimental interferograms as shown in Figs. $2 \mathrm{~b}$ and $3 \mathrm{~b}$, where the density gradients are very high. At least three pixels are necessary to visualize one fringe: a dark pixel between two white pixels. If the space between two fringes is smaller than one pixel, computed fringes will be displayed incorrectly. This problem can be avoided if the numerical image is created with a smaller number of fringes. See, for example, the image shown in Fig. 4a, where the two results match well with each other. Apart from this minor discrepancy, all the wave phenomena, such as the non-uniform flow expansion created at corners, the transmitted shock wave, the secondary shock waves, and the primary vortex loop, appear to be identical. From the direct comparison of these results obtained by viewing from three viewing directions, it can be concluded that the numerical solu-
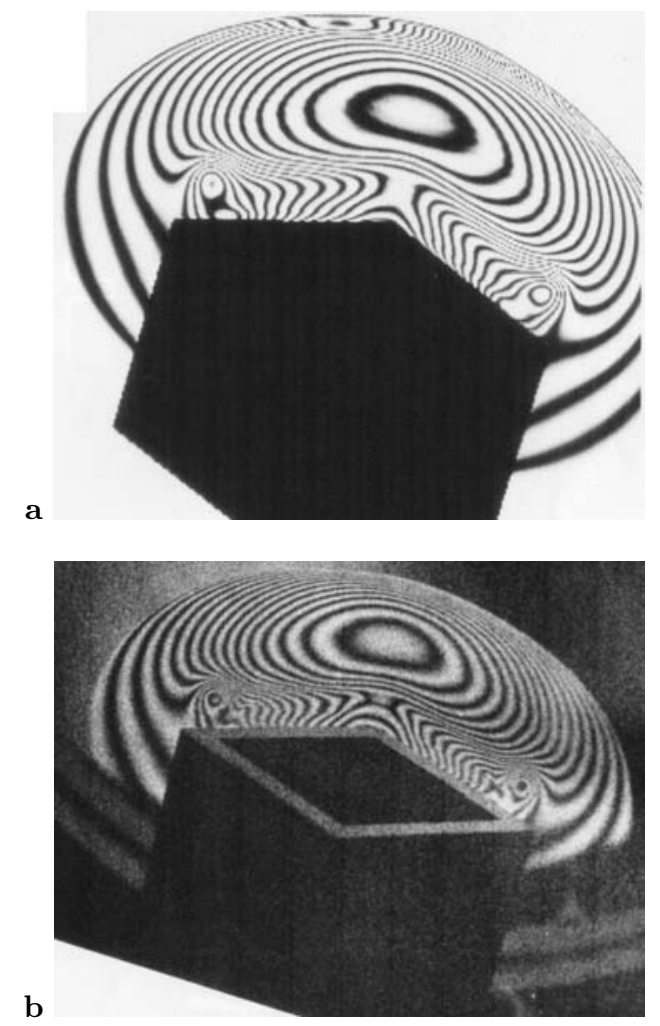

Fig. 4a,b. Axial views of a three-dimensional shock wave diffracting near the open end of a square shock tube at $M_{i}=$ 1.5. a Numerical interferogram; b experimental interferogram

tions are well validated and the numerical algorithms are acceptable for the present study.

It is obvious that for the validation of numerical simulations of such a flowfield with shock waves, a check on the numerical solutions with only a limited set of point measurements is not sufficient. A comparison between topological flow structures of the whole flowfield from both numerical and experimental results must be included in order to gain confidence on the numerical simulations. Moreover, since experimental results are not available in most of the cases under the CFD study, the validation cases have to be carefully selected so that the reliability of the numerical simulations could be demonstrated.

\section{Results and discussion}

In the following discussion, three major issues are especially emphasized in the present study. The first issue is the three-dimensional reflection of the transmitted shock wave, the pattern of which may vary with its Mach number. The second one is the spatial structure of the transmitted shock wave, which will transform from a planar to a three-dimensional one. The last is the possibility of verification of the observed wave phenomena with future experimental work, which is necessary because the flowfield under the CFD study is usually so complex that the experiments could be too difficult to be carried out by CFD researchers. By presenting numerical results that show the 
special features of the investigated flowfields which could be visualized and demonstrated experimentally, collaborations between experimentalists and CFD researchers can be promoted, and the research on flow mechanics will also benefit.

\subsection{Three-dimensional shock wave propagation at $M_{s}=1.5$}

The first case is the transmitted shock wave propagating at $M_{s}=1.5$ in the test section as shown in Fig. 1. The width of the chamber to that of the shock tube is $2: 1$. Sequential isopycnics of the numerical solutions are presented in Fig. 5, where four time sequential results show the evolution of the transmitted shock wave. The upper half of each result shows the density distribution in the diagonal plane and the lower half shows that in the mid-wall symmetrical plane.

Figure 5a shows the wave propagation at the instant shortly after the transmitted shock wave is discharged from the shock tube into the chamber. The shock wave diffraction at the shock tube exit is similar to that in the CFD validation case, as shown in Fig 4. Figure 5b shows the further propagation at the moment when the transmitted shock wave reflects from the chamber wall in the mid-wall symmetrical plane, and the resulting shock wave reflection pattern is a regular reflection. Meanwhile, the transmitted shock wave in the diagonal plane has not reached the chamber corners yet, being still in the diffracting phase. From these two figures, it is observed that the flow expansion created near the corners in the diagonal plane is stronger than in the mid-wall symmetrical plane. This is concluded by examining the density distribution behind the shock wave near the wall in both the upper half and the lower half of Fig. 5a, where the diffracting shock wave appears different in its intensity. Because of the nonuniform flow expansion, a secondary shock wave resulting from the locally-developed supersonic flow appears earlier near the primary vortex loop in the diagonal plane, as shown in Fig. 5b. The primary vortex loop developing from the shear layer originating from the edges of the shock tube does not move at a constant speed in the same direction due to the locally-developed supersonic flow. The part near the corner in the diagonal plane moves further downstream, as shown in the upper half of Fig. 5b, but the part in the mid-wall symmetrical plane moves more in the radial direction, as seen in the lower half of Fig. 5b. Therefore, the primary vortex loop, being of planar and square shape at its initial stage, is distorted and twisted into a three-dimensional one. The observation agrees well with the experiments reported by Abe and Takayama (1989) and Watanabe et al. (1992).

The shock wave reflection in the mid-wall symmetrical plane transforms from the regular to the simple Mach reflection in Fig. 5c. In the meantime, the regular reflection occurs near the chamber corners in the diagonal plane, so the transition from the regular to the simple Mach reflection exists spatially at the same time. Furthermore, in the upper half of Fig. 5 c, a weak shock wave is observable

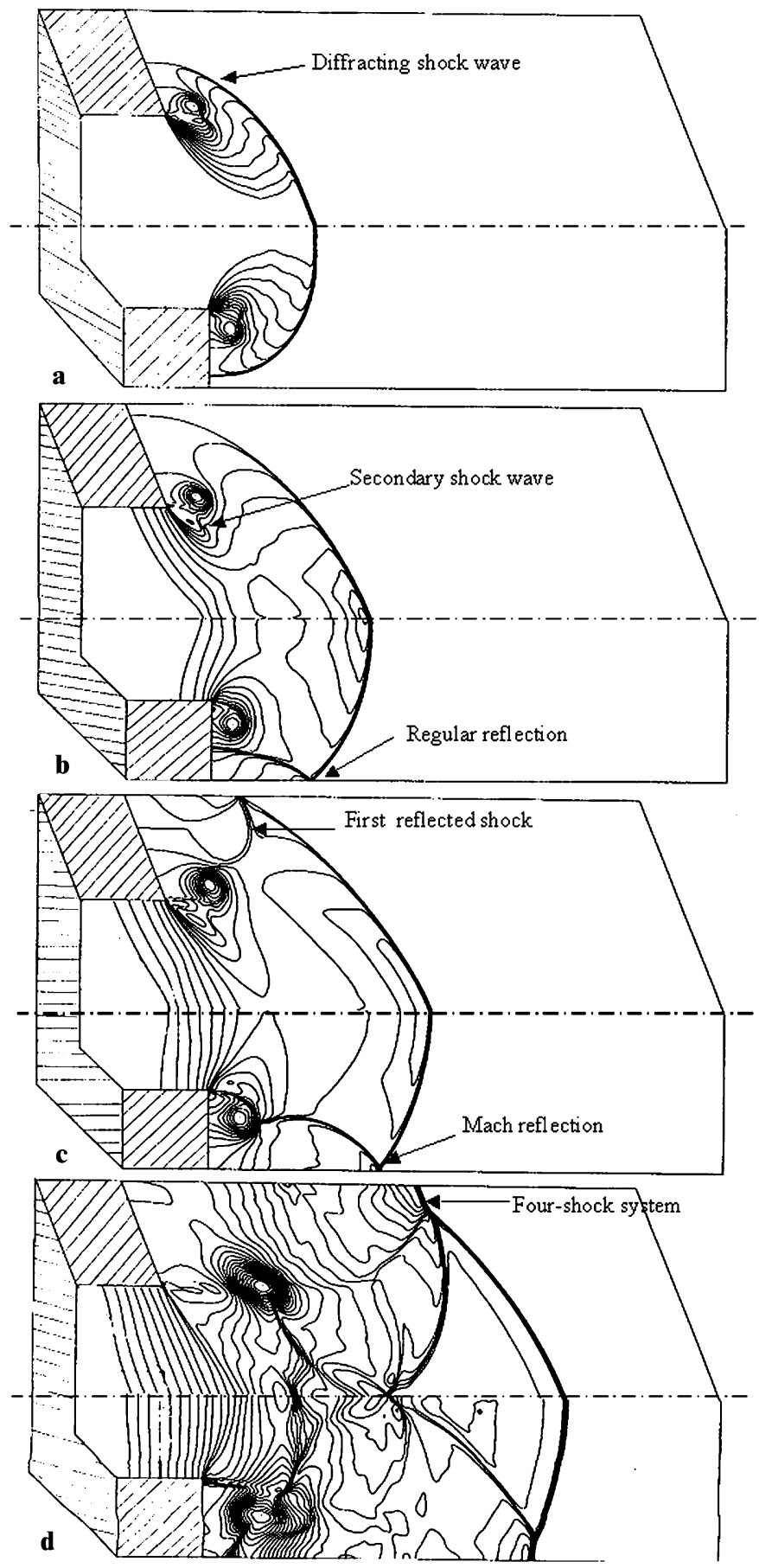

Fig. 5a-d. Sequential isopycnics of three-dimensional shock wave propagating in the large chamber at $M_{s}=1.5$, the upper half of each result showing the density in the diagonal plane and the lower half representing that in the mid-wall symmetrical plane

behind the reflected shock wave and looks unusual. One can imagine that when the reflected shock waves of regular reflection pattern merge in the diagonal plane from two sides of the chamber wall, a stronger reflected shock wave is created - referred to as the first reflected shock wave - and propagates faster. This leads to a mismatch 
of the reflection angle of the transmitted shock wave with its incident angle at the corner. Therefore, a new reflected shock wave behind the first one is generated with the reflection angle consistent with the incident angle, which is the so-called second reflected shock wave.

The first reflected shock wave interacts with the primary vortex loop in the mid-wall symmetrical plane, as shown in the lower half of Fig. 5c, which is a classical research topic of the shock-vortex interaction, but here the interaction is three-dimensional, which may results in more flow instability. The second reflected shock wave is clearly observable in Fig. 5d, and the Mach stem grows longer. At this time instant, a four-shock system develops, consisting of the transmitted shock wave, two reflected shock waves and a Mach stem. There are two triple-points in the system, but no shear layer is obviously observable here (in the diagonal plane) and also in the mid-wall symmetrical plane. The new wave system occurring in the diagonal plane in Fig. 5d is a typical Mach reflection but with an extra reflected shock wave. Considering the above explanation, this wave phenomenon may be observable only in three-dimensional cases because it develops from the collision of two shock wave reflection systems. In addition, the interaction of the second reflected shock wave with the primary vortex loop and the focusing of the first reflected shock wave on the axis of symmetry are observable in Fig. 5d. These processes will lead to the generation of vortices and turbulence, the research on which would require more complex physical and mathematic models, and is outside the scope of the present study.

\subsection{Three-dimensional shock wave propagation at $M_{s}=2.0$}

In order to clarify effects of the shock Mach number on shock wave reflection, the second case was carried out for a higher Mach number of $M_{s}=2.0$. Sequential isopycnics of its numerical results are presented in Fig. 6 with the same display style as used in Fig. 5.

Generally speaking, the wave phenomena observable here are similar to those observed in the first case, such as the non-uniform flow expansion, the locally-developed secondary shock wave, the shock/vortex interaction and the four-shock system. However, there are several differences worth pointing out. The first one is that the secondary shock wave in case 2 appears stronger than in the first case. By examining the density distributions shown in Figs. 5b and 6b, the secondary shock wave is readily observable in the mid-wall symmetrical plane in Fig. 6b, but not in Fig. 5b. This indicates that the local supersonic flow develops faster in case 2 due to the higher Mach number. The second difference is that the second reflected shock wave in the diagonal plane of case 2 is much more intense than that in case 1 . This is readily recognized by examining the upper half of Figs. $5 \mathrm{c}$ and $6 \mathrm{c}$, where the second reflected shock looks like compression waves in case 1 , but has developed into a shock wave in case 2 . The last difference is related to the development of the contact surface originating from the lower triple-point of the

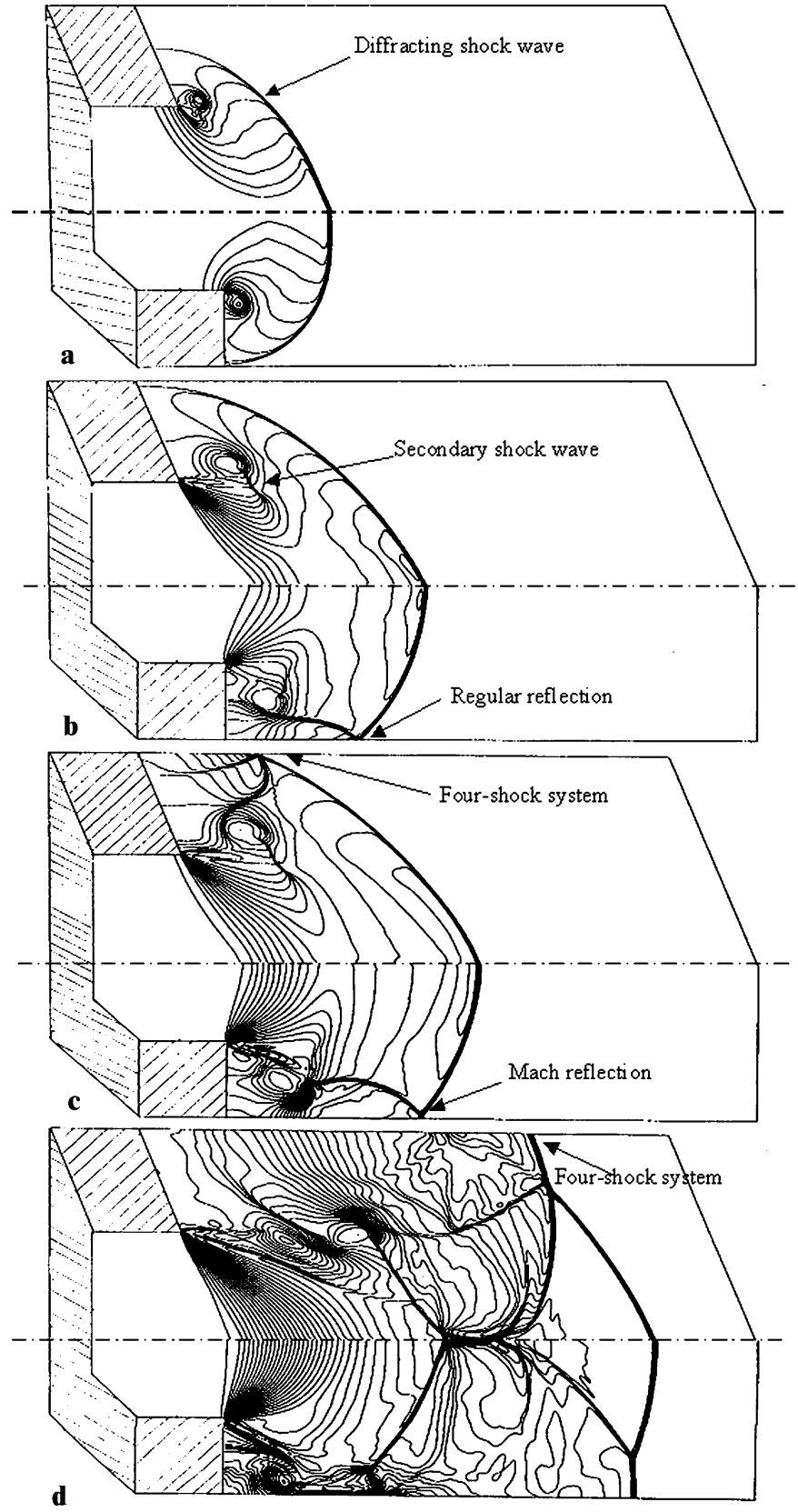

Fig. 6a-d. Sequential isopycnics of three-dimensional shock wave propagation in the large chamber at a Mach number of $M_{s}=2.0$, the upper half of each result showing the density in the diagonal plane and the lower half showing that in the mid-wall symmetrical plane

four-shock system, which is clearly observable in Fig. 6d, but hardly identifiable in Fig. 5d. Moreover, there is no contact surface appearing in the Mach reflection at the mid-wall symmetric plane in Fig. 5d, but it is quite observable in Fig. 6d. All of the processes discussed above seem to be due to the higher shock Mach number. The basic shock wave configuration of the three-dimensional shock wave propagation is not affected significantly, but more intense shock wave interactions are induced. 


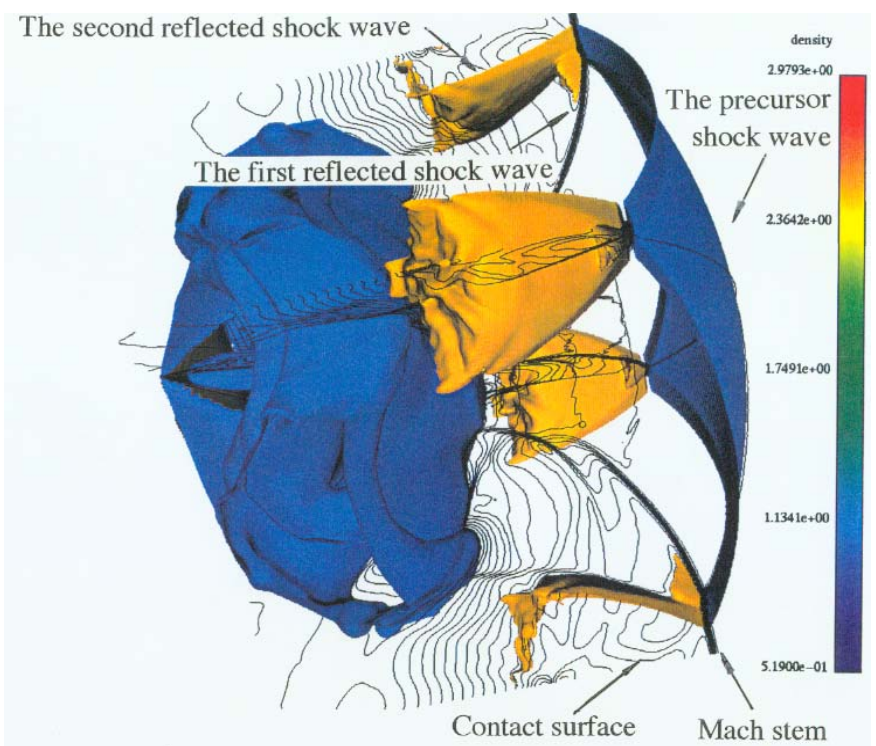

Fig. 7. Iso-density surfaces showing the three-dimensional configuration of the transmitted shock wave in the large square cross-section chamber at a Mach number of $M_{s}=2.0$

\subsection{Wave configuration of the transmitted shock wave at $M_{s}=\mathbf{2 . 0}$}

The three-dimensional configuration of the transmitted shock wave after its reflection from the chamber walls is presented in Fig. 7. The figure consists of the isopycnics in two diagonal planes and two iso-density surfaces. The first iso-surface of $\rho=1.12$ shows the transmitted shock wave, and the other of $\rho=2.3$ shows the reflected shock waves. The isopycnics in the two diagonal planes indicate the relation between the transmitted shock wave and the first or the second reflected shock wave.

From Fig. 7 it was observed that the transmitted shock wave in the central area of the large chamber still remains a spherical shape, the Mach stem near the chamber walls is curved forward having a three-dimensional configuration, and the part of the Mach stem near the chamber corners appears planar. It is also worth pointing out that the first reflected shock wave near the chamber corner induces a more intense density due to shock wave collision than that in the mid-wall symmetrical plane. Moreover, the density behind the second reflected shock wave in the diagonal plane near the chamber corner becomes even more intense due to the second shock wave reflection. This implies that the second reflected shock can catch up with the first one and the two triple-points will merge together. The isodensity surface behind the first reflected shock wave reveals the configuration of the second reflected shock wave, which confirms that the second shock wave is the reflection of the transmitted shock wave.

Figure 8 shows the configuration of the first reflected shock wave at the same instant as that shown in Fig. 7 . The figure, being similar to Fig. 7, also consists of the isopycnics in two diagonal planes and two iso-density surfaces: the iso-density surface of $\rho=1.12$ in Fig. 7 is replaced with one of $\rho=1.7$. The newly-added iso-density surface shows the configuration of the first reflected shock

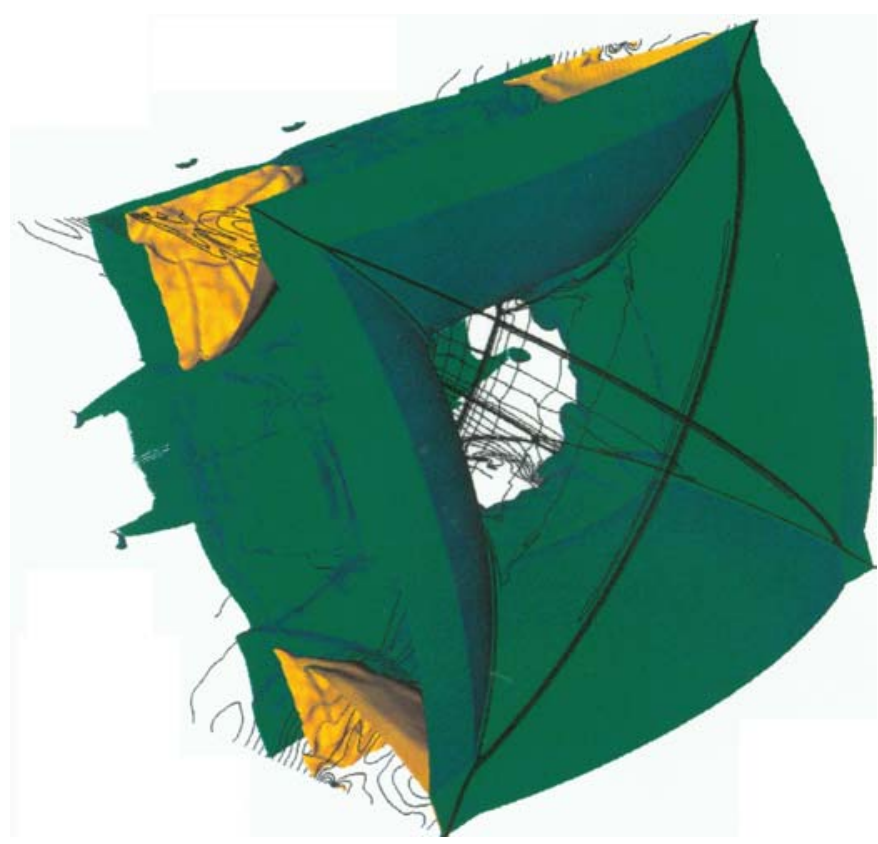

Fig. 8. Iso-density surfaces showing the three-dimensional configuration of the first reflected shock wave at a Mach number of $M_{s}=2.0$

wave and how it merges to generate an intense reflected shock at the chamber corners. The Mach stem of the transmitted shock wave is shown more clearly in Fig. 8, which supports the conclusion derived from Fig. 7. From the configuration of the first reflected shock wave, one can imagine that a strong jet will be generated later once the first shock wave merges on the axis of symmetry of the chamber. This will result in a square Mach disk shock that can catch up with the transmitted shock wave later and strengthens it.

From Figs. 7 and 8 , it can be concluded that the strengthened Mach stem due to the shock reflection from the chamber walls will propagate faster, catches up and overtakes the other part of the transmitted shock wave. On the other hand, the reflected shock wave will focus later in the centre area of the chamber, from which a Mach disk shock will develop. This shock can catch up with the transmitted shock wave in the centre area and strengthen it. In this way, the three-dimensional transmitted shock wave in a square chamber will transform back again into a planar one after it has propagated in the chamber for a certain distance.

\subsection{Computational interferograms}

Figures 7 and 8 show the complexity of the threedimensional shock wave propagation in a square chamber, from which the four-shock system appears to be an instinctive characteristic of the shock wave reflection. A question is whether it is possible to visualize the four-shock system with flow visualization techniques. If possible, what kind of flow images can be expected to see. A computational interferogram showing the corner view of the shock wave reflection is computed and presented in Fig. 9 to demonstrate the possibility. In addition, the numerical density 


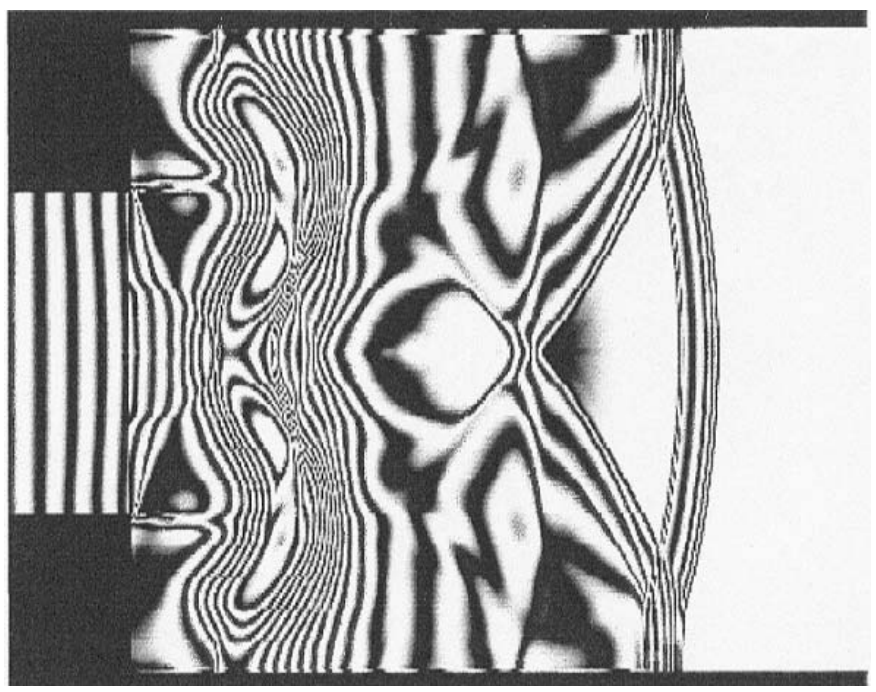

Fig. 9. Computational interferogram of the transmitted shock wave propagating at a Mach number of $M_{s}=1.5$ in the large chamber to demonstrate the possibility of visualizing experimentally the four-shock system, the corner view

distribution in the diagonal plane is also given in Fig. 10 as a reference, where isobars are plotted in its upper half and isopynics in the lower half. A very complicated fringe pattern appears in the Fig. 9 and looks quite different from the wave configuration shown in Fig. 10. As is well known, this fringe pattern results from the effect of integrating three-dimensional density distributions along the light paths. Fortunately, two discontinuities in the fringes behind the transmitted shock wave are still observable, which are located in the same positions as the first and the second reflected shock waves shown in Fig. 10. There is no doubt that the two discontinuities in Fig. 9 represent the two reflected shock waves. Thus, the four-shock system can be visualized experimentally, although the flow visualization images have to be carefully interpreted by considering the three-dimensional effects.

From the results shown in Figs. 9 and 10, one can see that it is possible to use flow visualization images for validating numerical solutions if such experimental results can be obtained. However, the flow visualization images may also lead to a confusion in the physics involved in the visualized flowfield because artificial effects have been introduced in the experiment due to the limitation of the current flow visualization techniques. Nowadays, most of the interesting problems are three-dimensional in nature and contain many transient phenomena. Investigations into such flowfields are very difficult, but important and useful. It may be too difficult to require experimental flow visualization to provide all the necessary information for understanding these complex flowfields, but it may be possible to circumvent this difficulty through interferometry, provided that interferogram images are clear enough to validate numerical solutions. In other words, experimental images of three-dimensional flowfields need only to provide data for validating numerical solutions. The numerical results thus validated, in return, would provide more useful information to interpret the complex flowfields.

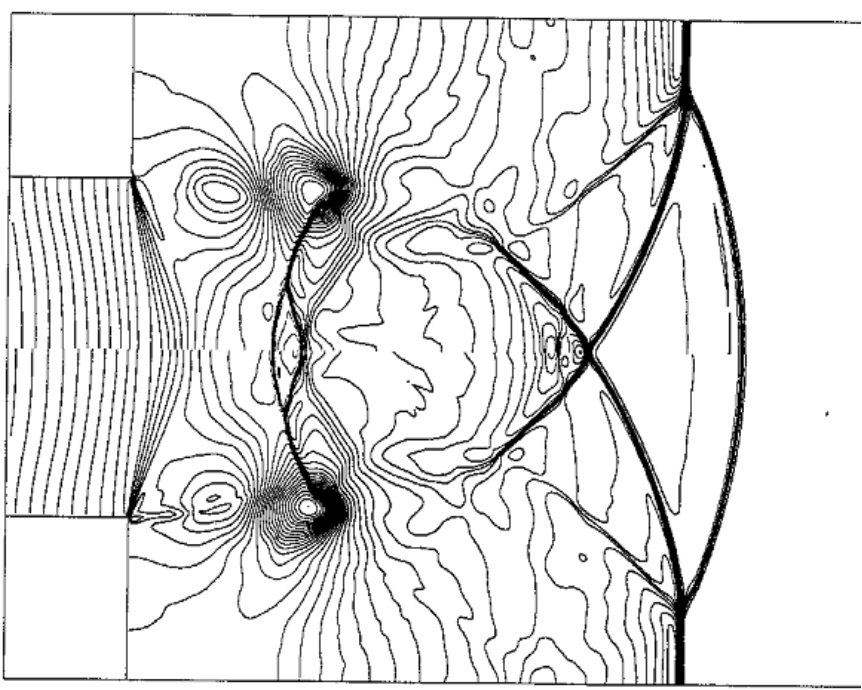

Fig. 10. Isobars (the upper half) and isopynics (the lower half) showing the numerical results in the diagonal plane of the transmitted shock wave propagating at a Mach number of $M_{s}=1.5$, presented as a reference to the computational interferogram

\section{Conclusions}

From the present numerical study, a four-shock system, consisting of a transmitted shock wave, two reflected shock waves and a Mach stem, is observed near the corner of the large chamber, which results from the interaction of reflected shock waves from the two sides of the chamber walls. This wave system can be a typical Mach reflection pattern in three-dimensional cases. The transition of shock wave reflection from the regular to the Mach reflection is also observed on the chamber walls at the same time in space due to the spherical shock wave reflecting from the square cross-section chamber. Moreover, the non-uniform expansion due to shock wave diffraction leads to the generation of a three-dimensional secondary shock wave resulting from locally-developed supersonic flows. Both the first and second reflected shock waves interact with the three-dimensional primary vortex loop and will lead to a distorted vortex loop as well as the generation of turbulence, the development of which are of great interest for further studies.

\section{References}

Abe A, Takayama K (1989) Shock wave diffraction from the open end of a shock tube. Proc of 17th Int Symp on Shock Wavews and Shock Tubes, 270-275

Abe A, Watanabe M, Suzuki K (1991) Three dimensional flow structure behind a shock wave discharged from a rectangular cross section shock tube. Takayama (ed) Proc 18th Int Symp Shock Waves, 209-212

Ben-Dor G (1992) Reflection of Shock Waves. Springer Verlag, New York

Casey TR, Takayama K (1991) Laser interferometry IV: computer-aided interferometry. Proc of Int Society for Optical Eng, 1553-1558 
Grönig H (1989) Past, present and future of shock wave focusing research. Proc of Int Workshop on Shock Wave Focusing, Sendai, Japan, 1-37

Hornung H (1986) Regular and Mach reflection of shock waves. Ann Rev Fluid Mech 18:33-58

Jiang Z (1993) Study on the finite difference theory and numerical methods of weak solution problems. Thesis, Peking University, Beijing, China

Jiang Z, Takayama K, Chen YS (1995) Dispersion conditions for non-oscillatory shock capturing schemes and its applications. Comp Fluid Dynamics J 2:137-150

Jiang Z, Onodera O, Takayama K (1999) Evolution of shock waves and the primary vortex loop discharged from a square cross-sectional tube, Shock Waves 9:1-10

Jiang Z, Takayama K (1999) An investigation into the validation of numerical solutions of complex flowfields. J Comp. Phys. 151:479-497
Meguro T, Takayama K, Onodera O (1997) Three-dimensional shock wave reflection over a corner of two intersecting wedges. Shock Waves 7:107-121

Skews BW (1967) The perturbed region behind a diffracting shock wave. J Fluid Mech. 29:705-719

Takayama K, Inoue O (1991) Shock wave diffraction over a 90 degree sharp corner: Poster presented at 18th ISSW. Shock Waves 1:301-312

Terao K (1984) Temperatures in imploding detonation waves. Jap J Appl Phys 23:27-33

Warming RF, Hyett BJ (1974) The modified equation approach to the stability and accuracy analysis of finitedifference methods. J Comp. Phys. 14:159-179

Watanabe M, Takayama K (1992) Visualization of shock waves phenomena by using double exposure holographic interferometry. J Visualization Soc Japan, 12:151-154 